\title{
Interaction entre les micromammifères et la production de semences forestières
}

\author{
J. P. VINCENT \\ Laboratoire de la Faune Sauvage. Centre national de Recherches zootechniques, I.N.R.A. \\ 78350 Jouy-en-Josas.
}

\begin{abstract}
Résumé
L'interaction entre les microrongeurs forestiers et la production de semences a été étudiée dans les 3 forêts de Bellême (Orne), Fontainebleau (Seine-et-Marne) et Villers-Cotterêts (Aisne). L'action des petits rongeurs se manifeste par l'attaque des graines tombées au sol et des jeunes semis. Les mesures effectuées au moyen d'enclos montrent qu'au moins 50 p. 100 des semences disparaissent sous l'action des rongeurs en période de forte production. L'intensité de ce phénomène varie en fonction de l'importance de la chute des graines et du niveau des populations des 2 espèces principales rencontrées : le Mulot (Apodemus sp.), et le Campagnol roussâtre (Clethrionomys glareolus).

En retour, une production abondante de graines forestières agit sur les populations de microrongeurs. On observe une reproduction hivernale et une amélioration de la survie des jeunes. Ces 2 facteurs déferminent une augmentation rapide des densités conduisant à des pics hivernaux de populałion.
\end{abstract}

La régénération naturelle des forêts est soumise à de nombreux aléas : irrégularité de la production de semences, apparition de graines avortées, destruction par les insectes, rongeurs, oiseaux ef mauvaises conditions rencontrées au sol. Ce sont les relations existant entre les populations de microrongeurs et la production de semence qui retiendront notre attention ici et nous étudierons les 2 aspects suivants : 1 . Action des rongeurs sur la production (prédation) ; 2 . Influence de la production de graines sur les populations de rongeurs.

\section{Małériel et méthodes}

Les résultats que nous présentons proviennent d'une étude du peuplement de microrongeurs forestiers entreprise à propos des problèmes posés par la régénération naturelle des forêts de plaine. Ces recherches se sont étendues sur 3 années (1972 à 1975) et ont été menées dans 3 forêts du bassin parisien (Fontainebleau - Seine et Marne -, Bellême - Orne - et Villers-Cotterêts - Aisne).

La méthode classique du piégeage en ligne (Spiłz, 1Súg; Spitz et al. 1974) nous a permis de suivre l'évolution des populations des 2 espèces principales : le Mulot (Apodemus sp.) et le Campagnol roussâtre (Clethrionomys glareolus). Tous les 2 mois, 6 à 9 lignes nous fournissent un échantillon représentatif du peuplement. Divers para- 
mètres démographiques (âge, état sexuel, production de jeunes) sont étudiés par l'autopsie des Captures.

L'influence des rongeurs sur la production des semences se manifeste soit par la consommation des graines tombées au sol soit par l'attaque des jeunes semis. Nous avons essayé de quantifier la disparition des graines que nous avons assimilé à une consommation avec donc un risque de surestimation.

Ces essais mettaient en œuvre des batteries d'enclos protégeant une zone de 1 mètre carré de surface contre l'attaque de tous les animaux à l'exception des petits rongeurs. Des cages sans fond en grillage à maille carrée de 1 centimètre sont posées sur le sol et ensemencées avec des glands ou des faines à des densités voisines de celle que l'on rencontre dans la station. Dans la pratique, nous avons pris 10 semences/mètre carré les années où la production était faible et 100 /mètre carré lorsqu'elle était bonne.

La comparaison est faite avec des zones témoins de même surface et ensemencées à la même densité, mais laissées libres d'accès.

Un comptage mensuel permet de suivre l'évolution de la disparition des graines dans les témoins et les enclos de protection contre les rongeurs. La mise en place des batteries s'effectue au moment de la chute maxima des semences.

Les essais se sont déroulés dans des chênaies (forêts de Bellême et Fontainebleau) et des hêtraies (Villers-Cotterêts et Fontainebleau). Les parcelles choisies sont toutes en cours de régénération, au stade de l'ensemencement ou des coupes secondaires. II s'agit donc de peuplements entrouverts où le sous-étage est absent. La strate herbccée est celle de la chenaie acidiphile à ronces abondantes à Fontainebleau, et de la chenaie sessiliflore à houx pour Bellême.

Signalons que la technique s'est révélée peu appropriée pour l'étude de la disparition des faines dont les faibles dimensions rendaient les comptages très aléatoires. Aussi ne présenterons nous ici que les résultats concernant les glands.

\section{Résultats}

1. Etude de la consommation des glands par les rongeurs

Nous avons effectué 3 campagnes de mesure :

- automne-hiver 1972-1973 à Fontainebleau uniquement où 8 batteries furent installées (1 témoin +1 enclos rongeur par batterie) ;

- automne-hiver 1973-1974 à Fontainebleau et Bellême où 16 batteries ont été utilisées (1 témoin +1 enclos rongeurs par batterie) ;

- automne-hiver 1974-1975 à Bellême avec 3 batteries (2 témoins et 5 enclos rongeurs par batterie).

A titre indicatif nous donnons ci-après quelques chiffres sur l'importance de la glandée à Bellême les années 1972-1973 et 1974 :

1972 : presque rien ;

1973 : 50 à 90 glands/mètre carré, assez bonne ;

1974 : 25 à 50 glands/mètre carré, petite glandée partielle.

C'est la campagne d'automne-hiver 1973-1974 qui a donné lieu au plus grand nombre de relevés, aussi présenterons-nous d'abord ces résultats. Ils regroupent les 
données recueillies à Bellême ef Fontainebleau lors d'une année où la production de glands fut très bonne.

On se reportera au tableau 1 et à la figure 1 pour le détail des résultats.

\section{TABLEAU 1}

Disparition moyenne des glands en fonction du temps

(73-74 Fontainebleau + Bellême)

Mean disappearance of acorns in time

(73-74 Fontainebleau $\dashv$ - Bellême)

\begin{tabular}{|c|c|c|c|c|c|c|}
\hline \multirow[b]{2}{*}{ Enclos (Enclosure) } & \multicolumn{6}{|c|}{ Date (Date) } \\
\hline & $\begin{array}{l}\text { Oct. } \\
\text { (Oct.) }\end{array}$ & $\begin{array}{l}\text { Nov. } \\
\text { (Nov.) }\end{array}$ & $\begin{array}{l}\text { Déc. } \\
\text { (Déc.) }\end{array}$ & $\begin{array}{c}\text { Janv. } \\
\text { (Jan.) }\end{array}$ & $\begin{array}{l}\text { Fév. } \\
\text { (Feb.) }\end{array}$ & $\begin{array}{c}\text { Mai } \\
\text { (May) }\end{array}$ \\
\hline Témoin (Control). & 5,5 & 22,7 & 34,6 & 45,5 & 48,6 & 61,2 \\
\hline Rongeur (Rodent) ............ & 1,5 & 16,8 & 21,0 & 31,8 & 32,9 & 39,4 \\
\hline to rodents) $\ldots \ldots \ldots \ldots \ldots \ldots \ldots$ & 27 & 74 & 60 & 69 & 67 & 65 \\
\hline
\end{tabular}

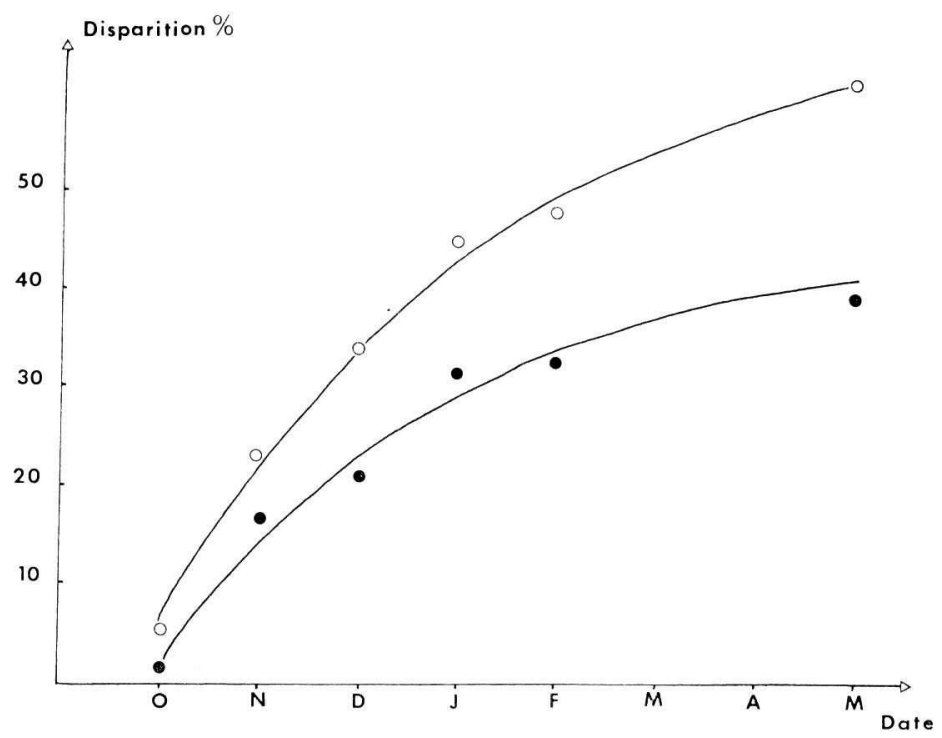

FIG. 1. - Évolution de la disparition de glands dans les enclos (Fontainebleau + Bellême - 73-74)

$$
\begin{aligned}
& 0 \text { - } \\
& - \text { - Rémoins } \\
& \text { - Rongeurs }
\end{aligned}
$$

FIG. 1. - Progression of acorn disappearance in enclosures (Fontainebleau + Bellême 73-74) ;

Disappearance p. 100.

$$
\text { - O Controls. }
$$


Plusieurs points nous paraissent importants :

a) la disparition dans les placettes témoins atteint environ 60 p. 100 de l'effectif initial,

b) cette valeur est atteinte après un délai d'environ 8 mois,

c) la courbe de disparition des glands (fig. 1) dans les témoins a une allure semblable à celle des enclos rongeurs, mais cette dernière présente un palier en début de printemps. A cette époque, la part qui revient aux rongeurs dans la disparition est d'environ 65 p. 100.

\section{TABLEAU 2}

Disparition moyenne des glands en fonction du temps

72-73 Fontainebleau

Mean disappearance of acorns in time

(72-73 Fontainebleau)

\begin{tabular}{|c|c|c|c|c|}
\hline \multirow[b]{2}{*}{ Enclos (Enclosure) } & \multicolumn{4}{|c|}{ Date (Date) } \\
\hline & $\begin{array}{l}\text { Déc. } \\
\text { (Déc.) }\end{array}$ & $\begin{array}{l}\text { Janv. } \\
\text { (Jan.) }\end{array}$ & $\begin{array}{l}\text { Fév. } \\
\text { (Feb.) }\end{array}$ & $\begin{array}{l}\text { Mars } \\
\text { (Mar.) }\end{array}$ \\
\hline Témoin (Control). ............... & 25 & 40 & 63 & 65 \\
\hline Rongeur (Rodent)..... & 25 & 40 & 50 & 51 \\
\hline $\begin{array}{l}\text { P. } 100 \text { de disparition dû aux ron- } \\
\text { geurs (p. } 100 \text { of disappearance due } \\
\text { to rodents) } \ldots \ldots \ldots \ldots \ldots \ldots \ldots \ldots\end{array}$ & 100 & 100 & 79,3 & 78,4 \\
\hline
\end{tabular}

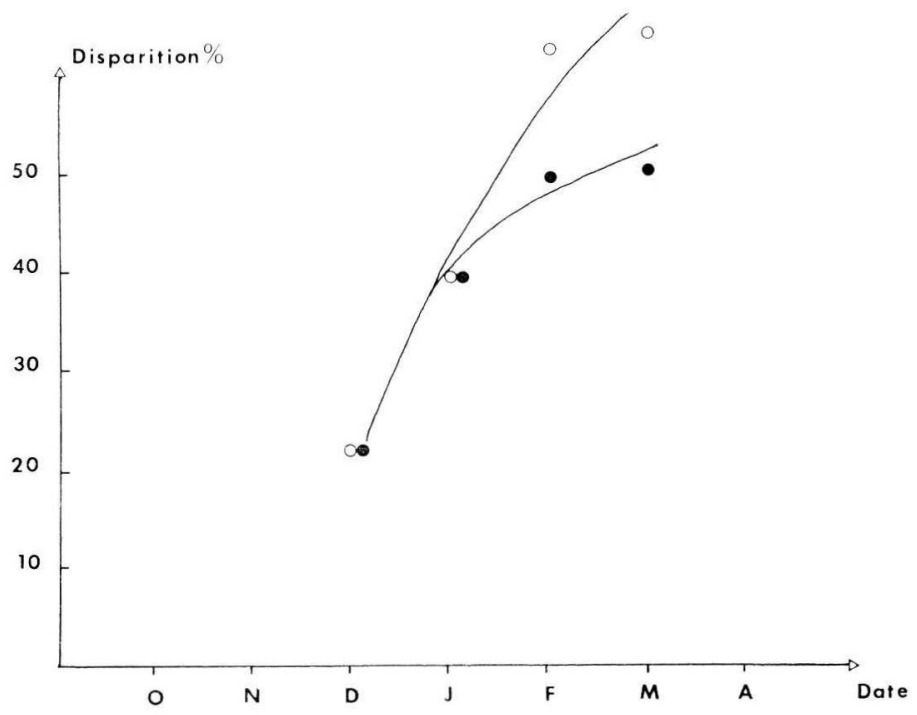

FIG. 2. - Evolution de la disparition des glands dans les enclos à Fontainebleau en 72-73.

$$
\begin{aligned}
& \bigcirc-O \text { Témoins } \\
& - \text { - Rongeurs }
\end{aligned}
$$

FIG. 2. - Progression of acorn disappearance in enclosures (Fontainebleau + Bellême 73-74) ; 
Les résultats obtenus lors de la campagne de l'automne-hiver 1972-1973 (où la glandée était peu importante) (tabl. 2, fig. 2) diffèrent quelque peu des précédents.

En 4 mois, le stock initial de glands diminue de 65 p. 100 et les rongeurs y ont participé pour 78 p. 100. La vitesse de disparition, donc l'intensité de l'attaque est, en outre, bien plus forte que précédemment. Enfin au cours de l'expérience d'automnehiver 1974-1975 c'est en 10 jours que 90 p. 100 des glands déposés dans les enclos disparaissent sous l'action pratiquement des seuls rongeurs (99,1 p. 100).

Ces résultats sont regroupés dans le tableau 3 où figurent, à côté des divers paramètres mesurés, les densités moyennes du peuplement de rongeurs pour la durée de l'expérience. Les 3 années étudiées ont connu des glandées différentes. II semble cependant que pour des intensités de glandées voisines, l'attaque est d'autant plus forte et rapide que la densité de rongeurs est plus forte.

TABLEAU 3

Comparaison des trois années de relevés

Results of three years compared

\begin{tabular}{ccccc}
\hline & $\begin{array}{c}\text { Densité moyenne } \\
\text { de rongeurs } \\
\text { (Mean rodent } \\
\text { density) }\end{array}$ & $\begin{array}{c}\text { Part des rongeurs } \\
\text { dans la disparition } \\
\text { (p. } 100 \text { disappearance } \\
\text { due to rodents) }\end{array}$ & $\begin{array}{c}\text { Etendue de la } \\
\text { période d'attaque } \\
\text { (Length of attack } \\
\text { period) }\end{array}$ & $\begin{array}{c}\text { Intensité } \\
\text { de la glandée } \\
\text { (Acorn crop size) }\end{array}$ \\
\hline $72-73$ & $73,9 / 10$ ha & 78,4 p. 100 & 4 mois & + \\
$73-74$ & $111,5 / 10$ ha & 65 p. 100 & 8 mois & +++ \\
$74-75$ & $201 / 10$ ha & 99,1 p. 100 & 10 jours & ++ \\
\hline
\end{tabular}

Les micromammifères apparaissent donc comme un des prédateurs les plus importants des semences après la chute. Nous avons mesuré la disparition des gland's et, dans cette première approche, nous l'avons assimilé à une consommation qui ne laissait aucune chance à la graine de se développer ultérieurement. Mais on est en droit de supposer que toutes les disparitions observées n'impliquent pas obligatoirement une destruction. Des glands transportés et non consommés dans l'immédiat pourraient germer s'ils sont oubliés par les animaux. Cependant nous n'avons jamais observé de « greniers » de Mulots ou de Campagnols dans la nature, et la littérature consultée n'en signale pas non plus. Aussi pensons-nous que les graines qui disparaissent sont perdues pour la régénération.

Les valeurs de disparition indiquées sont des moyennes calculées à partir d'un certain nombre de batteries. Mais des variations assez considérables existent d'une batterie à l'autre selon le site choisi, car la population de rongeurs n'est pas uniformément répartie. Les impératifs matériels et techniques n'ont pas permis d'augmenter le nombre des batteries qui aurait sans doute amélioré l'échantillonnage.

Sur le plan pratique, la nécessité de recompter régulièrement les mêmes enclos nous a conduit à préparer les zones ensemencées en les débarrassant du couvert de litière. Cela a pu retentir sur le comportement des animaux au cours de la recherche de leur nourriture, mais les témoins étant traités de la même façon les comparaisons restent valables entre témoins et enclos rongeurs. 
Dans une étude sur le régime alimentaire du Campagnol roussâtre selon la densité de population, Holisova (1971) signale que 38,8 p.100 du volume total de nourriture consommée par cette espèce est constitué par des graines pendant les périodes de faible densité. En volume total, ce régime est plus abondant en graine que ne l'est celui de l'espèce en période de fortes densités. Et c'est en automne et hiver que les grains et fruits d'essences ligneuses prédominent dans le régime.

Pour notre part, compte tenu des imperfections de la technique utilisée, on peut affirmer que les rongeurs sont responsables d'au moins 50 p. 100 de la disparition des glands après leur chute. Nous n'avons pas de résultats valables concernant les faines, mais on est en droit de supposer qu'ils sont du même ordre de grandeur.

\section{Influence de la production de semences forestières sur les populations de rongeurs}

Nous étudierons dans ce chapitre le problème inverse de celui traité précédemment, à savoir l'action d'une glandée ou d'une fainée sur le développement des populations de microrongeurs. Nous considérerons pour cela 3 paramètres : la reproduction, l'évolution des densités et la dynamique des effectifs.

\section{a) Etude de la reproduction.}

L'époque et la durée de la saison de reproduction sont portées en traits pleins $\epsilon \dagger$ pointillés horizontaux sur la figure $n^{\circ} 3$. L'intensité (p. 100 de femelles actives) est représentée par les petits traits verticaux. Les flèches verticoles accompagnées des lettres $F$ et $G$ symbolisent la présence d'une glandée ou d'une fainée.

Une variabilité extrême se dessine quant à la durée $\epsilon$ t'époque de la reproduction. Schématiquement la situation peut se résumer ainsi : les années à glandée cu fainée on note la présence d'une reproduction hivernale plus ou moins intense. Cela prend la forme soit d'une prolongation de la saison d'été jusqu'au printemps suivant (ou à la limite jusqu'à l'été suivant, comme ce fut le cas à Bellême en 1973-1974) soit du redémarrage de la reproduction en automne après un arrêt momentatné (Villers-Cotterêts, en 1974-1975). On remarquera de même une différence significative de la tailla des portées, chez le Mulot, entre le printemps et l'automne : respectivement 3,8 et 4,8 petits par portée en moyenne pour les deux époques. Une bonne chute de graines stimule donc la production de jeunes.

b) Evolution des densités.

L'étude de la figure 3 montre que les densités subissent un cycle annuel qui les fait passer par des maximums en été ou en automne-hiver selon les années. Les minimums ont une position plus constante, vers le mois d'avril d'une façon générale. A peu de chose près, l'évolution est synchrone chez les 2 espèces.

Les années où la production de semences cst faible ou nulle, comme ce fut le cas à Fontainebleau en 1972 et 1974, les maximums de dr nsité se situent en été et les valeurs atteintes sont relativement modestes. La chute consécutive des effectifs est lente, étalée dans le temps, ce qui donne à la courbe une allure $\in n$ cloche. L'hiver est une période de décroissance progressive de la densité jusqu'au printemps où un minimum est atteint en avril (Fontainebleau 1974-1975, Bellêmie 1974-1975).

En revanche lorsque la production de graines est bonne, les densités présentent un maximum d'hiver. Celui-ci est en général accentué et prend la forme d'un pic. 
Villers-Cotterêtéts
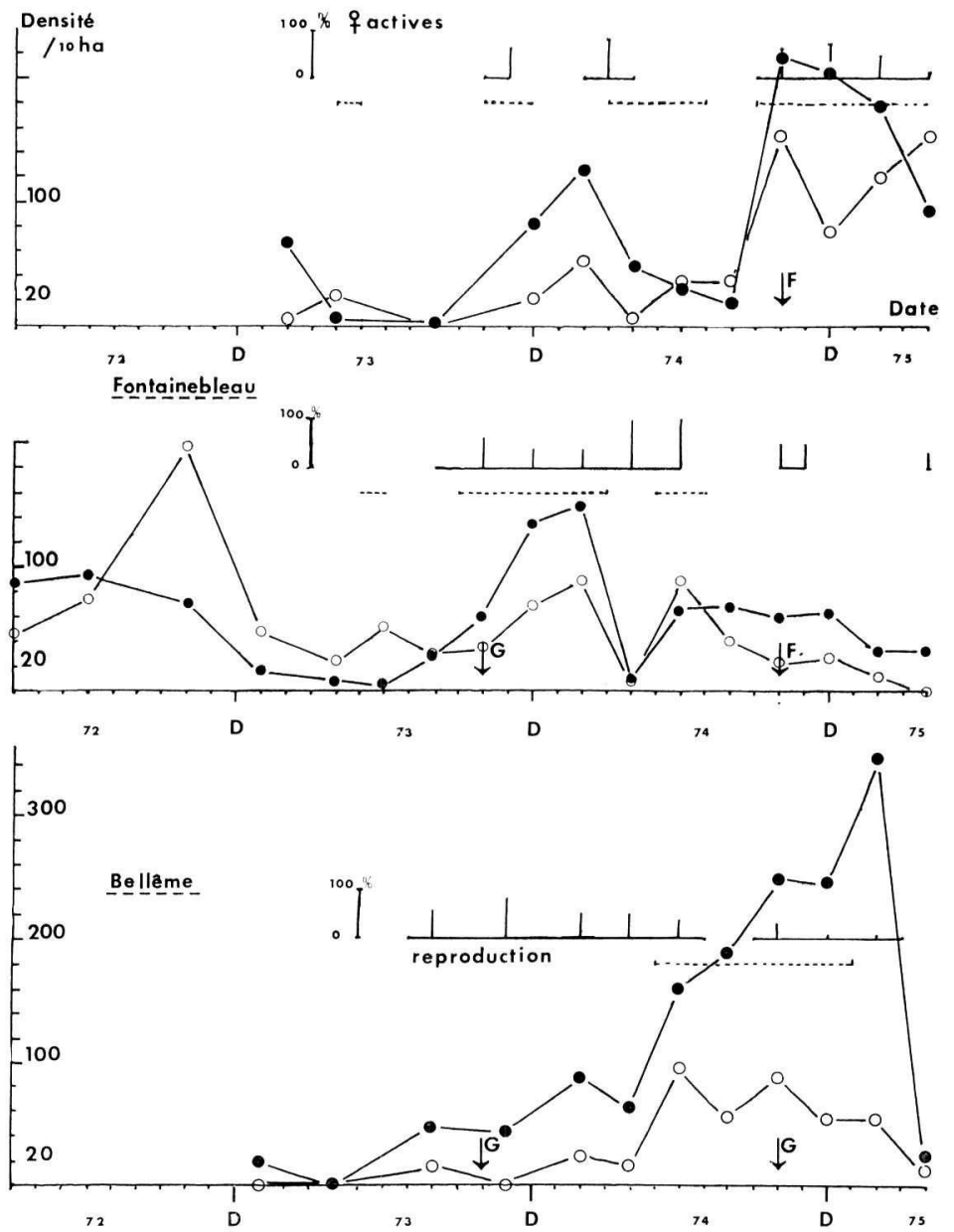

FIG. 3. - Evolution des densités de rongeurs en fonction du temps.

Progression of rodent density in time.

$$
\begin{aligned}
& - \text { - Apodemus sp. } \\
& 0 \text { - } 0 \text { Clethrionomys glareolus. }
\end{aligned}
$$

C'est une augmentation brutale et intense du niveau de population (Villers-Cotterêts, hiver 1974-1975, Fontainebleau 1973-1974, Bellême hiver 1974-1975).

Soulignons le cas de Bellême où 2 années de suite la glandée fut bonne. Les densités, parties d'un niveau presque nul au printemps 1973, commencent une croissance régulière et intense sur une période de plus de 1 an. Seule une légère dépression en avril 1974 rappelle les minimums habituellement rencontrés à cette époque. II est intéressant également de noter l'écroulement brutal des effectifs entre février et avril 1975 qui fait passer les densités de plus de 340 animaux pour 10 hectares à 20. 
c) Etude de la dynamique des effectifs.

La dynamique des effectifs d'une population de microrongeurs apparaît comme le bilan des entrées et des sorties d'animaux dans cette population c'est-à-dire entre la natalité et la mortalité ou plus exactement natalité + immigration et mortalité + émigration.

Nous présentons dans la figure 4 l'évolution des quotients bimensuels de mortalité chez le Mulot, calculés à partir de diagramme de Lexis, selon la méthode utilisée par Spitz dans son étude de la démographie du Campagnol des champs (Spitz, 1972). Compte tenu de la durée limitée de l'étude, ce graphique ne couvre que l'année 1974 et le débuł 1975.

Si l'on considère la situation à Bellême, on note qu'à peu de chose près les mortalités sont en augmentation régulière entre aoûł 1974 et avril 1975. Mais elles touchent principalement les animaux les plus âgés de sorte que l'apport de jeunes dans la population, dû à une intense reproduction n'est contrebalancé que par la disparition d'animaux âgés et peu nombreux. Il se produit donc un phénomène d'accumulation qui tend à gonfler les effectifs. Par la suite, les mortalités atteignent pour toutes les classes (les jeunes y compris) des valeurs élevées. C'est à ce moment que l'on observe l'effondrement des effectifs.

II apparaît donc que l'augmentation continue de la population est le fait d'une meilleure survie des jeunes par rapport aux animaux plus âgés, pour autant que la reproduction se maintienne à un niveau élevé. Par la suite, l'apport de jeunes cessant progressivement avec la diminution puis l'arrêt de la reproduction, conjointement à l'augmentation générale de la mortalité, un déséquilibre se produit entre les entrées et les sorties et la chute des densités s'amorce.

Les phénomènes sont de même nature à Villers-Cotterêts. La montée rapide des Effectifs entre août et octobre 1974 esł le fait d'une mortalité générale faible et d'une natalité importante. Le recrutement des jeunes n'est contrebalancé par aucune sortie, et la population croît. Dans les mois qui suivent, bien que la natalité se maintienne à un niveau élevé, les effectifs entament une diminution qui ira en s'accentuant. On remarque en effet que les mortalités ont augmenté considérablement et les entrées ne compensent pas les pertes puisque les nouveaux venus sont éliminés et les anciens continuent de disparaître. A partir de février, l'activité reproductrice qui s'éteint prècipite le déclin des densités.

En automne 1974 à Fontainebleau la glandée fut très peu abondante et la reprise de la reproduction éphémère (octobre-novembre). La chute de la mortalité de la classe no 3 (animaux de 2 à 4 mois) entre octobre et décembre explique le palier de densité que l'on observe pendant cette période. A partir de décembre toutes les mortalités sont en hausse, la reproduction arrêtée, les densités diminuent.

Si la dynamique des effectifs intègre les 2 phénomènes, natalité et mortalité, il semble que finalement c'est le facteur de mortalité qui joue le rôle prépondérant selon l'âge des individus touchés.

Lors d'une période de recrutement important de jeunes même la mortalité des adultes n'empêche pas l'augmentation de population. Au contraire si les jeunes sont éliminés très vite, en dépit de l'apport qu'ils représentent, les effectifs peuvent diminuer même en période de reproduction. 


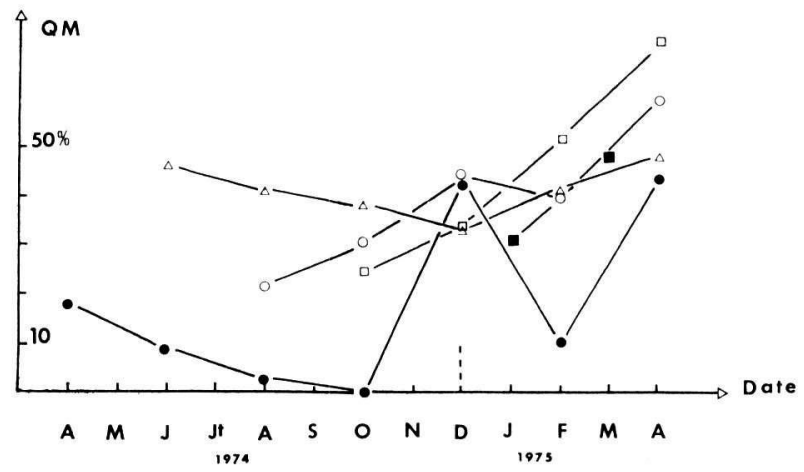

FIG. 4a. - Forêt de Bellême. FIG. 4a. - Bellême forest.

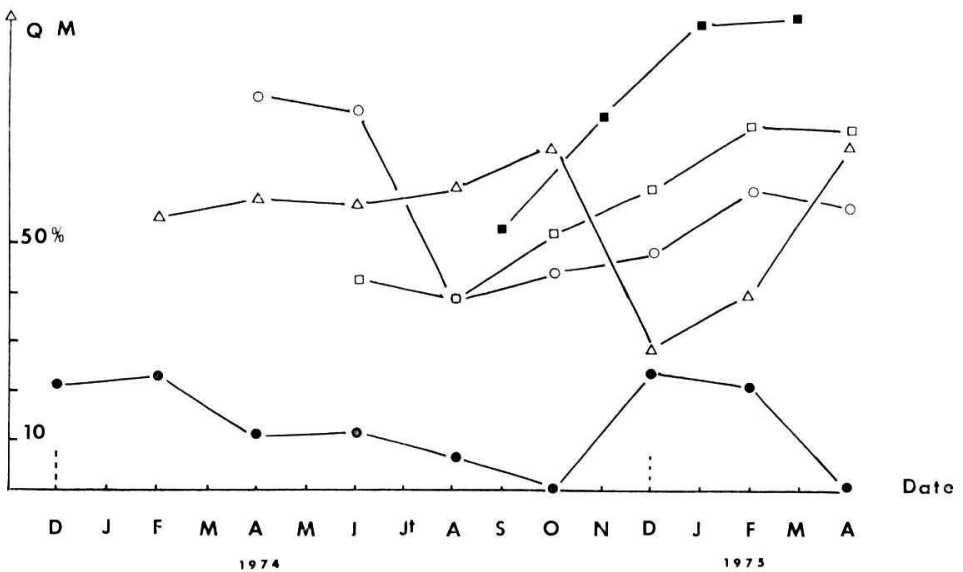

FIG. 4b. - Forêt de Fontainebleau. FIG. 4b. - Fontainebleau forest.

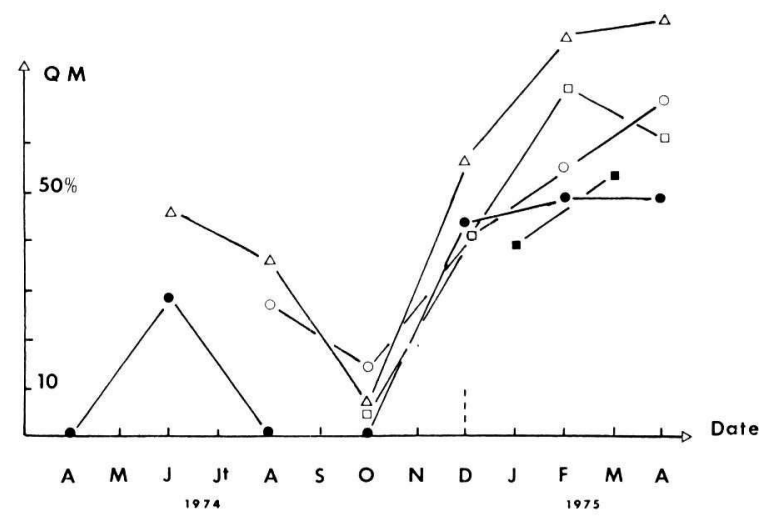

FIG. 4c. - Forêt de Villers-Cotterets. FIG. 4c. - Villers-Cotterets forest.

FIG. 4. - Evolution des Quotients bimensuels de mortalité chez le Mulot.

Progression of bi-monthly mortality quotients in the field-mouse.

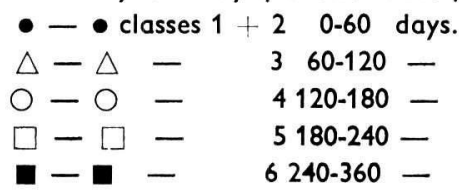


La production de semences forestières retentit sur les populations de rongeurs de 2 façons : on observe une stimulation de la reproduction qui reprend ou se continue pour la période hivernale et une meilleure survie des jeunes. Ces deux facteurs jouent dans le même sens pour déterminer le gonflement des effectifs, mais c'est en dernière analyse, le facteur mortalité qui préside aux variations des densités selon son intensité ef l'âge des animaux concernés.

Des phénomènes analogues ont été signalés dans la littérature. Le Louarn (1972) trouvait un résultat semblable chez le Mulot lors d'une fainée abondante.

De nombreux auteurs citent une reproduction hivernale (Turcek (1956) ; Smyth (1960) ; Zejda (1962) ; Newson (1963) ; Birkan (1968) qu'ils attribuent à des conditions favorables du milieu, température, précipitation et surtout disponibilité en nourriture. Des essais d'apport artificiels de nourriture aboutissent de la même façon à une reproduction hivernale (Flowerdew (1972) ; Andrzejewski (1974). Watts (1969) trouve que chez le Mulot l'augmentation de la densité automnale est due à l'amélioration de la survie des jeunes.

\section{Conclusion}

Une liaison assez étroite se dessine entre le peuplement de rongeurs et la production de semences forestières. En tant que consommateurs primaires, ces derniers utilisent, pour leur nourriture, toute production végétale disponible et profitent au mieux de la manne que représente une chute abondante de glands ou de faines. II s'ensuit une stimulation de la reproduction à une époque où habituellement elle a cessé et une amélioration de la survie des jeunes. La densité de population augmente alors plus ou moins brutalement et dans des proportions variables liées à l'abondance de la chute des graines. La consommation des semences s'accentue en conséquence au point d'entraîner l'épuisement des ressources alimentaires qui en retour détermine une diminution des effectifs. Une telle situation s'est présentée à Bellême où 2 années consécutives de glandée ont conduit la population de rongeurs à des densités très élevées. Cette saturation du milieu a rapidement abouti à la pénurie et à l'écroulement très brutal des densités en avril 1975 où les mortalités de toutes les classes d'âge étaient élevées.

Pour expliquer de telles variations brutales de densité, certains auteurs (Evans, 1942 ; Lidicker, 1962 ; Trojan, 1965) avancent l'hypothèse d'une dispersion des animaux à la recherche de milieux plus favorables. Cette question a souvent été posée, notamment pour le Mulot, dont on soupçonne la capacité d'effectuer des déplacements à grandes distances. Des essais de marquage effectués à Fontainebleau semblent confirmer cette capacité, mais aucune donnée précise ne permet de faire la part de la mortalité et de la dispersion dans le phénomène observé.

La réussite d'une glandée ou d'une fainée n'a donc pas d'influence durable sur une population de rongeurs. Elle stimule temporairement son développement qui se trouve bloqué dès que l'apport alimentaire fourni est épuisé. 


\section{Summary \\ Interaction between small mammals and forest seed production}

The interaction between small forest rodents and seed production is studied in the 3 forests of Bellême (Orne), Fontainebleau (Seine et Marne) and Villers-Cotterêts (Aisne). Small rodent action consists of attacking seeds fallen to the ground and young seedlings. Measurements taken within an enclosure show that at least 50 p. 100 of the seeds disappear due to rodent action in a high production period. The intensity of this phenomenon varies with the number of seeds fallen and the population level of the two main species found, the long-tailed field-mouse (Apodemus sp.) and the bank vole (Clethrionomys glaerolus).

On the other hand, abundant production of forest seeds effects small mammal populations ; winter reproduction is observed and survival of the young is better. These 2 factors determine the rapid density increase causing winter population peaks.

\section{Références bibliographiques}

ANDRZEJEWSKI R., 1974.The influence of the forage basis upon the dynamics in the number of the population of Clethrio nomys glareolus schreb (Rodentia). Theriological congress Moscow, 6-12 juin 1974, p. 27.

B!RKAN M., 1968. Répartition écologique et dynamique des populations d'Apodemus sylvaticus et Clethrionomys glareolus en pinèdes à Rambouillet. La Terre ef la Vie 1968-3, 231-273.

EVANS F. C., 1942. Studies of small mammal population Bacley wood Berkshire Journal of Animal Ecology, vol. 11, no 2, pp. 182-197, nov. 1942.

FLOWERDEW J. R., 1972. The effect of supplementary food on a population of wood mice (Apodemus sylvaticus). J. Anim. Ecol., 41, 553-566, oct. 1972.

HOLISOVA V., 1971. The food of Clethrionomys glareolus at different population densities. Acta Sc. Nat. Brno, 5 (11) : 1-43.

LE LOUARN H. et SCHMITT A., 1972. Relations observées entre la production de faines et la dynamique de populations du Mulot, Apodemus sylvaticus L. en forêt de Fontainebleau. Ann. Sci. Forest. 30 (2) : 205-214.

LIDICKER W. Z., 1962. Emigration as a possible mechanism permitting the regulation of population density below carrying capacity. Ann. Nat., 96, 29-33.

NEWSON R., 1963. Difference in number, reproduction and survival between two neighboring populations of bank voles (Clethrionomys glareolus). Ecology, 44, 110-120.

SMYTH H., 1960. Winter breeding in wood land mice, Apodemus sylvaticus, and voles Clethrionomys glareolus and Microtus agrestis, near Oxford. J. Anim. Ecol., 35, 471-485.

SPITZ F., 1969. L'échantillonnage des populations de petits Mammifères. In Problèmes d'écologie: I'échantillonnage des peuplements animaux en milieux terrestres, 153-188. Masson, Paris.

SPITZ F., LE LOUARN H., POULET A., DASSONVILLE B., 1974. Standardisalion des piègeages en ligne pour quelques espèces de rongeurs. La Terre ef la Vie, 28, 564-578.

SPITZ F., 1972. Démographie du campagnol des champs (Microtus arvalis Pallas) en Vendée Thèse de Doctorat ès Sciences naturelles. Université de Paris VI. Paris 1972.

TROJAN P., 1965. Intra population relations and regulation of numbers in small forest rodents ekol. pol. (Ser. A.), 13., 143-68.

TURCEK F. J., 1956. Quantitative experiments on the consumption of tree seeds by mice of the species Apodemus flavicollis. Archivum Societatis zoologicae Botanicae fennicae « Vanome ", $10,1$.

WATTS C. H. S., 1969. The regulation of wood mouse (Apodemus sylvaticus) Numbers in Witham woods, Berkshire. J. of an. ecol., 38, 285-304 ; June 1969.

ZEJDA, 1962. Winter breeding in the bank vole, Clethrionomys glareolus Schreber, 1870, Zool. Listy 25, 309-321. 